\title{
Erratum to: Chapter 55 in Information Science and Applications (ICISA) 2016
}

\section{Erratum to:}

Chapter 55 in: K.J. Kim and N. Joukov (eds.)

Information Science and Applications (ICISA) 2016, DOI: 10.1007/978-981-10-0557-2_55

The last author name should be changed to "Maneesha V. Ramesh"

The online version of the original chapter can be found under DOI: 10.1007/978-981-10-0557-2_55 